\title{
La nécropole romaine de Porta Nocera à Pompéi : le secteur 26 OS
}

La campagne 2014

William Van Andringa, Thomas Creissen et Henri Duday

\author{
(2) OpenEdition \\ Journals \\ Édition électronique \\ URL : http://journals.openedition.org/cefr/1352 \\ DOI : $10.4000 /$ cefr.1352 \\ ISSN : 2282-5703 \\ Éditeur \\ École française de Rome \\ Référence électronique \\ William Van Andringa, Thomas Creissen et Henri Duday, «La nécropole romaine de Porta Nocera à \\ Pompéi : \\ le secteur 26 OS », Chronique des activités archéologiques de l'École française de Rome [En ligne], Les \\ cités vésuviennes, mis en ligne le 04 mai 2015, consulté le 22 novembre 2019. URL : http:// \\ journals.openedition.org/cefr/1352; DOI : 10.4000/cefr.1352
}

Ce document a été généré automatiquement le 22 novembre 2019.

(c) École française de Rome 


\section{La nécropole romaine de Porta Nocera à Pompéi : le secteur 26 OS}

La campagne 2014

William Van Andringa, Thomas Creissen et Henri Duday

\section{NOTE DE L'ÉDITEUR}

Rédacteur de la chronique : Thomas Creissen avec la collaboration de William Van Andringa

Auteurs du rapport : Marie-Josée Ancel (Archeodunum), Hélène Barrand Emam (Antea), Philippe Brunner, Cécilia Cammas (Inrap), Marianna Castiglione (Scuola Normale, Pisa), Marie-Caroline Charbonnier (Inrap), Carole Chevalier, Thomas Creissen (Éveha international), Franck Decanter (Inrap), Henri Duday (CNRS, Université de Bordeaux), Johannes Laiho, Tuija Lind, Adrien Malignas (université de Montpellier), MalebogoDob Mvimi, Clarisse Parra-Prieto, Véronique Zech-Matterne (CNRS), Géraldine Sachau-Carcel (université de Bordeaux), William Van Andringa (université de Lille 3).

\section{NOTE DE L'AUTEUR}

La campagne 2014 du programme de recherche PORTA NOCERA 2 s'est déroulée du $1^{\mathrm{er}}$ au 30 septembre 2014. Nous tenons à remercier particulièrement le Prof. Soprintendente Massimo Osanna et la Dott.ssa Grete Stefani, directrice du site de Pompéi, qui ont rendu possible la mise en œuvre de ce programme d'étude ainsi que Catherine Virlouvet, directrice de l'École française de Rome, Stéphane Bourdin, directeur des études pour l'Antiquité, sans oublier Giulia Cirenei, secrétaire pour l'Antiquité.

Sur place, l'opération de fouille a bénéficié de l'aide précieuse, dans la coordination des travaux, de la Dott.ssa Annalisa Capurso, funzionario archeologo, et de l'Assistente 
Ingeniere Vicenzo Sabini. Notre gratitude va également aux institutions qui portent le programme, l'École française de Rome, l'université de Lille 3 et le laboratoire Halma ainsi qu'Éveha International.

1 La campagne de 2014 a été la première du nouveau projet de recherche consacré à la nécropole de la Porta Nocera. Elle s'est déroulée au cours du mois de septembre ${ }^{1}$. Les fouilles se sont concentrées sur un secteur assez limité d'environ $60 \mathrm{~m}^{2}$. Il s'agit d'un terre-plein vierge de toute construction situé entre l'enclos 23 OS à l'est et le monument funéraire 27 OS à l'ouest (fig. 1).

Fig. 1 - Plan de la zone étudiée : le secteur 26 OS entre les mausolées 23 OS à l'est et 27 OS à l'ouest.

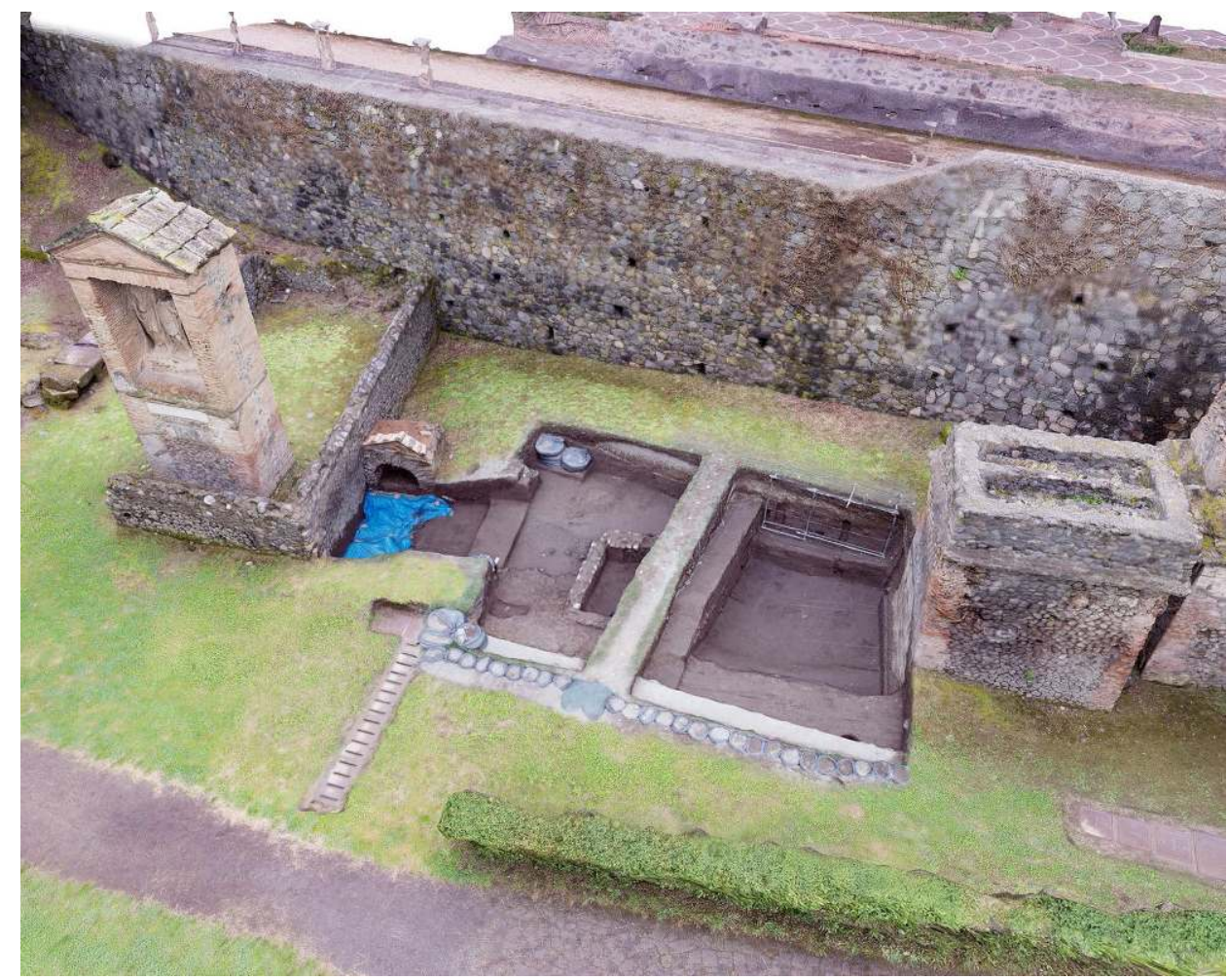

(c) Porta Nocera 2 (J. Laiho). Sur concession du Ministero per i Beni e le attività culturali - Soprintendenza archeologica di Pompei. Toute reproduction, par quelque moyen que ce soit, reste interdite.

Dans le but d'élaborer une stratégie d'étude adéquate pour les prochaines années, le principal objectif visait à évaluer le potentiel archéologique du secteur, et notamment à caractériser au mieux les niveaux funéraires (présence de tombes, de bûchers...). Cette campagne a par ailleurs été l'occasion d'expérimenter de nouvelles méthodes d'enregistrement, en particulier avec un large recours à la photogrammétrie. Celle-ci a été utilisée afin de documenter les différentes étapes de la fouille. Les principales découvertes ont elles-aussi été modélisées. Plus généralement, c'est toute la zone étudiée qui a été traitée afin d'obtenir un modèle numérique de tout ce secteur de la ville.

3 Eu égard à la configuration de la fouille, il a été nécessaire d'isoler trois secteurs qui ne sont pas tous parfaitement contigus : 26 Ouest, 26 Est et $25 \mathrm{C}$ (fig. 2). Cet éparpillement empêche d'établir systématiquement de strictes équivalences stratigraphiques. 
Fig. 2 - Plan des secteurs de fouille.

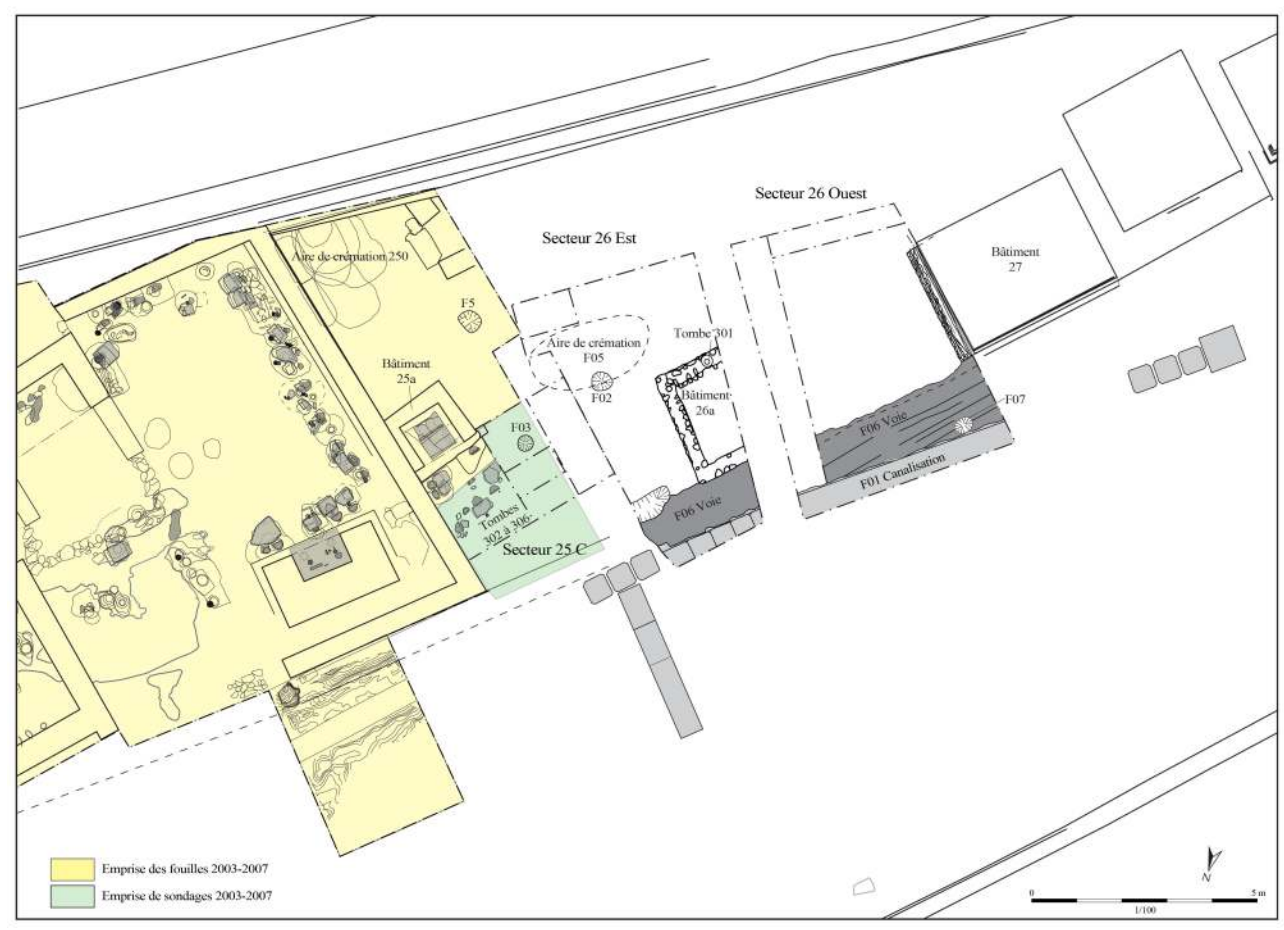

(c) Porta Nocera 2 (C. Chevalier, J. Laiho et T. Lind). Sur concession du Ministero per i Beni e le attività culturali - Soprintendenza archeologica di Pompei. Toute reproduction, par quelque moyen que ce soit, reste interdite.

Par ailleurs, l'étude du matériel a tout juste été entamée au cours de cette campagne : les datations ne sont pas encore bien établies. Pour toutes ces raisons, les propositions de phasage formulées ci-après sont bien évidemment sujettes à révision.

\section{Les plus anciennes traces d'occupations : des espaces funéraires en bordure d'une voie}

5 La plus ancienne trace d'occupation du secteur est pour l'instant documentée par le grand monument funéraire 27 OS qui en constitue la limite occidentale. Dégagé en 1956 à l'occasion des fouilles dirigées par A. Maiuri, ce monument est daté de la seconde moitié $\mathrm{du} \mathrm{I}^{\mathrm{er}}$ siècle av. J.-C., et plus vraisemblablement du troisième quart de ce siècle.

6 Les fouilles ont permis de dégager environ $1,70 \mathrm{~m}$ d'élévation supplémentaire pour ce monument qui mesurait donc plus de $5 \mathrm{~m}$ de haut. La construction repose sur un imposant podium surmonté d'une plinthe moulurée, l'ensemble des parements étant recouvert d'un enduit plus ou moins bien conservé. Les niveaux d'occupations contemporains de l'érection du monument n'ont pas été atteints. Un sondage profond a simplement permis d'identifier un niveau de sol qui pourrait être contemporain de son «utilisation» (il est situé contre le parement est). Cependant, l'observation est restée trop ponctuelle pour que l'on puisse en dire davantage. Ce niveau apparaît à environ $8,05 \mathrm{~m}$ NGF.

7 À l'opposé de ce monument, en partie orientale du secteur fouillé, les investigations archéologiques réalisées entre 2005 et 2007 sur le site de Porta Nocera avaient permis de 
mettre en évidence l'existence d'une concession funéraire antérieure à la niche 25A, c'està-dire au monument funéraire de Castricia Prisca qui sera évoqué plus bas. La partie sommitale de deux stèles était alors apparue (tombes 104 et 105, correspondant aux SEP 302 et 303 dans le nouveau programme). La reprise des fouilles a permis d'identifier trois autres tombes (fig. 3).

Fig. 3 - Relevé en plan du secteur $25 \mathrm{C}$.

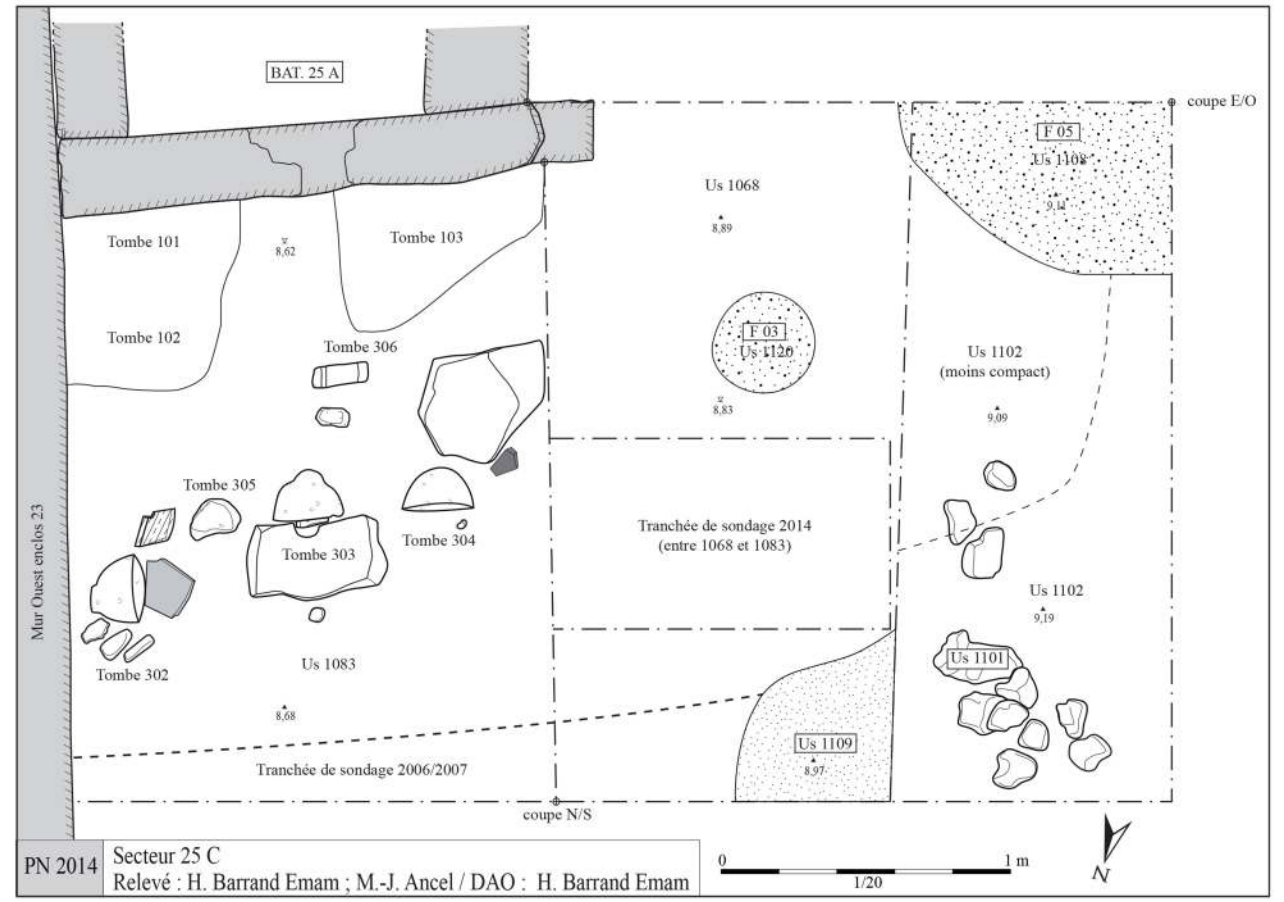

(c) Porta Nocera 2 (M.-J. Ancel, H. Barrand Emam). Sur concession du Ministero per i Beni e le attività culturali - Soprintendenza archeologica di Pompei. Toute reproduction, par quelque moyen que ce soit, reste interdite.

8 Toutes sont pourvues d'un système de marquage: des columelles pour quatre d'entre elles (SEP 302 à 305), une stèle en pierre à la partie sommitale simplement arrondie pour la cinquième (SEP 306). Des plaques de scellement sont associées à certaines d'entre elles. Ces tombes apparaissent à une altitude moyenne de 8,70 m NGF.

Faute de temps, cet ensemble funéraire a simplement été dégagé : il sera fouillé lors de la campagne de 2015. Les éléments de datation sont donc rares. Certaines tombes sont alignées le long du mur ouest de l'enclos 23 , mur dont les travaux antérieurs ont pu déterminer qu'il était postérieur à $20 \mathrm{av}$. J.-C. Les tombes ne sont donc pas antérieures à la période augustéenne. L'étude préliminaire du matériel invite à resserrer la fourchette aux premières décennies de notre ère.

En partie centrale du secteur étudié, les vestiges d'un monument inédit 26a, en grande partie ruiné, sont apparus (fig. 4). Il s'agit d'une construction quadrangulaire dont le plan n'est pas entièrement connu, puisqu'il passe pour partie sous la berme conservée afin de faciliter les circulations. 
Fig. 4 - Photographie verticale du monument funéraire 26a, secteur 26 OS Est.

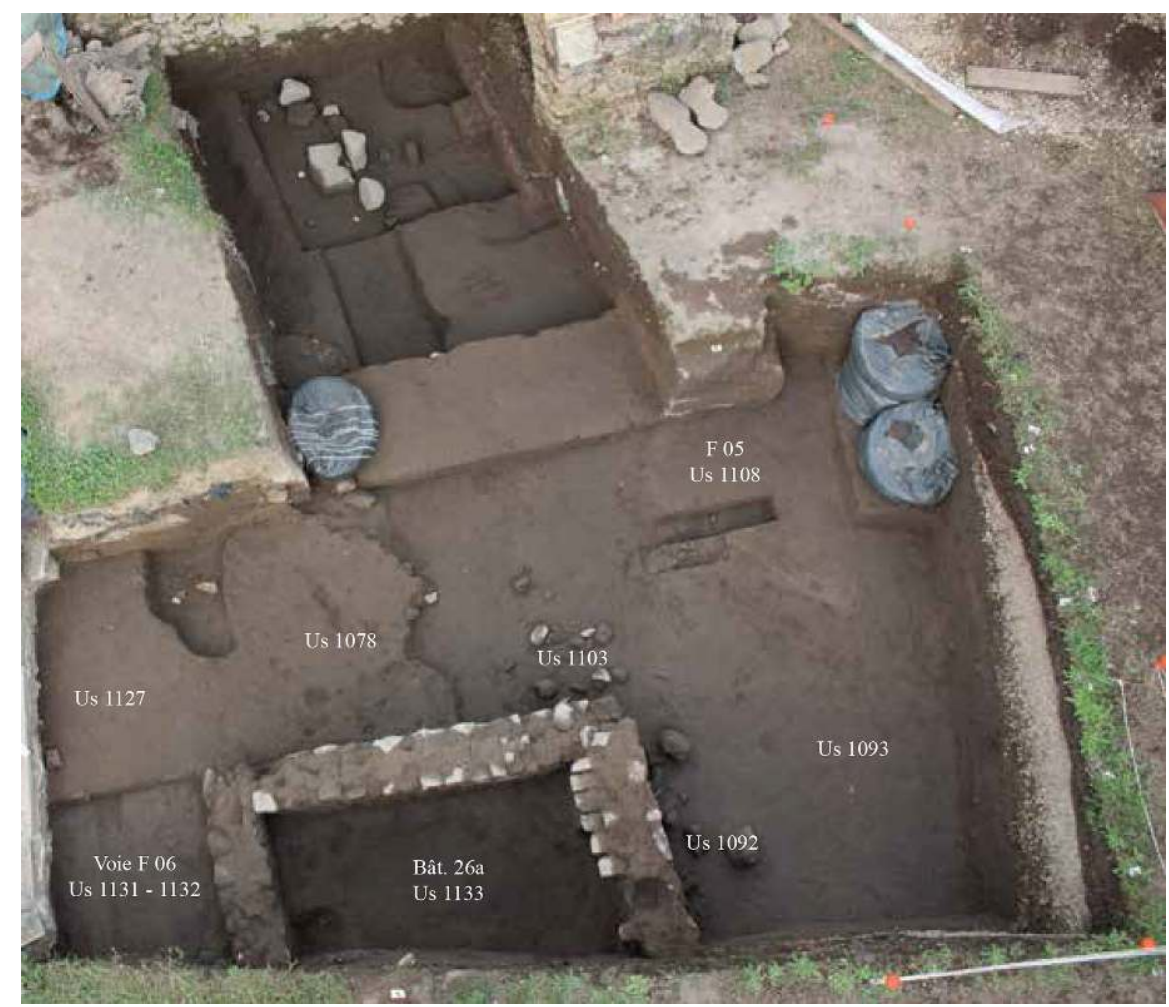

(c) Porta Nocera 2 (G. Sachau-Carcel). Sur concession du Ministero per i Beni e le attività culturali Soprintendenza archeologica di Pompei. Toute reproduction, par quelque moyen que ce soit, reste interdite.

Le mur oriental, intégralement dégagé, mesure 2,60 $\mathrm{m}$ de long. Les retours nord et sud font au moins 1,60 $\mathrm{m}$ de long. Tous les murs sont épais d'une trentaine de centimètres, et ils sont à peu près construits de la même manière. Plusieurs aménagements spécifiques sont associés au mur sud. Une assise de blocs en légère saillie forme comme une corniche au sein de cette maçonnerie. Surtout, à la même hauteur, une tombe est installée au cœur de la maçonnerie : l'empreinte du contenant funéraire - une urne en verre - est encore bien visible. Il a été directement scellé dans le mortier de construction et appartient donc au projet initial. Seule une partie de cette tombe était préservée, l'essentiel ayant été détruit par la suite (SEP 301). L'examen des restes osseux permet d'y reconnaître la tombe d'un adulte plutôt jeune et extrêmement gracile. Au milieu des ossements humains, plusieurs fragments d'os finement ouvragés ont été découverts : ils appartiennent vraisemblablement au lit funéraire sur lequel le défunt a été brûlé (fig. 5). 
Fig. 5 - Tombe 301, élément de placage du lit funéraire en forme d'aile.

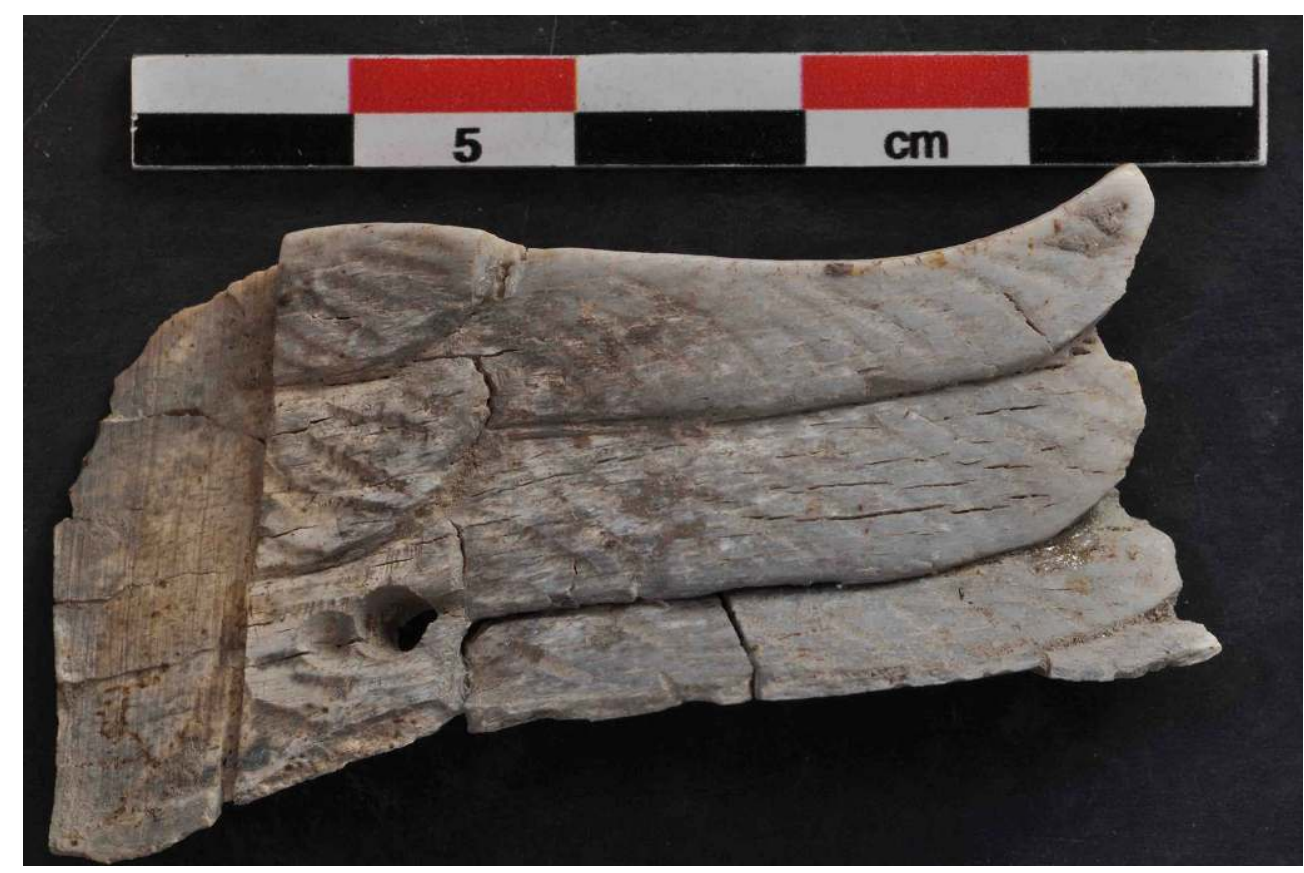

(c) Porta Nocera 2 (H. Duday). Sur concession du Ministero per i Beni e le attività culturali -

Soprintendenza archeologica di Pompei. Toute reproduction, par quelque moyen que ce soit, reste interdite.

12 L'élévation maximale observée est de $90 \mathrm{~cm}$, mais les niveaux de sol contemporains de la construction comme de l'utilisation de ce monument n'ont pas été atteints lors de cette campagne, aussi bien à l'intérieur qu'à l'extérieur. Par conséquent, la datation du monument reste pour l'instant incertaine. En l'état actuel des connaissances, l'hypothèse de son appartenance à la période augustéenne est privilégiée.

13 Tous ces aménagements - enclos funéraire, construction ruinée 26a ou imposant monument funéraire 27 OS - étaient plus ou moins directement liés à une importante voie qui avait la même orientation que celle encore visible plus à l'est. Cette voie, notamment associée à la Porte de Nocera, est restée en usage jusqu'à l'éruption de 79 apr. J.-C.

14 Pour l'essentiel, seules les recharges les plus tardives ont été exposées ou en partie fouillées en 2014. Les investigations archéologiques permettent toutefois de mettre en évidence une évolution très prononcée dans les rapports qu'entretenait cette voie avec les secteurs environnants (fig. 6 et 7). 
Fig. 6 - Secteur 26 OS Ouest, évolution de la voie F06 et de ses abords selon la coupe Nord, planche $1 / 2$.

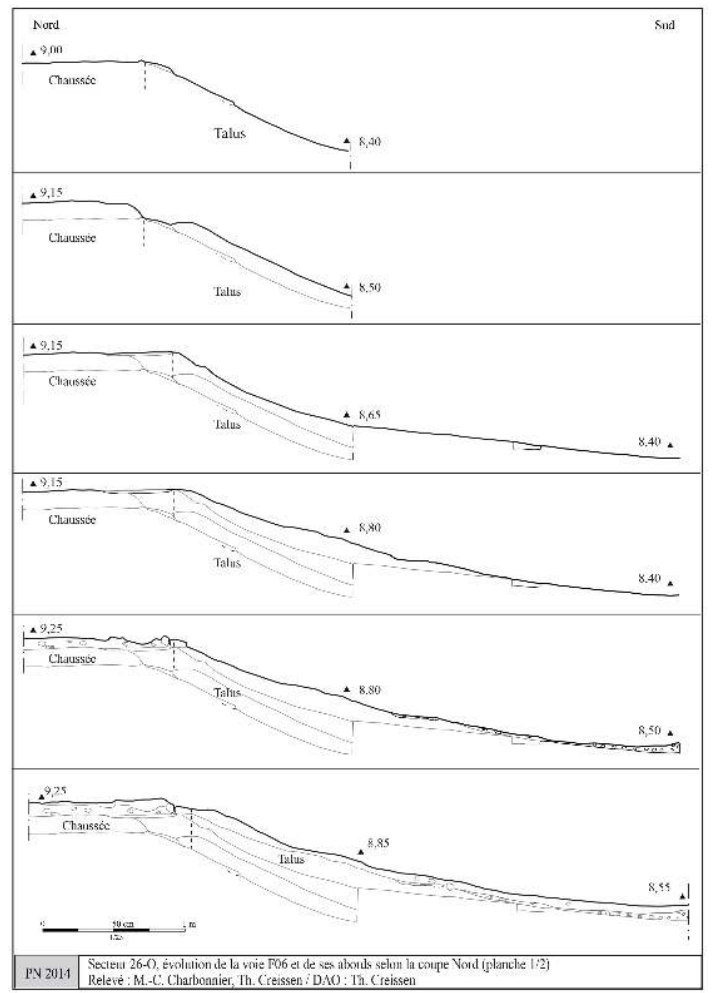

(c) Porta Nocera 2 (M.-C. Charbonnier, Th. Creissen). Sur concession du Ministero per i Beni e le attività culturali - Soprintendenza archeologica di Pompei. Toute reproduction, par quelque moyen que ce soit, reste interdite. 
Fig. 7 - Secteur 26 OS Ouest, évolution de la voie F06 et de ses abords selon la coupe Nord, planche $2 / 2$.

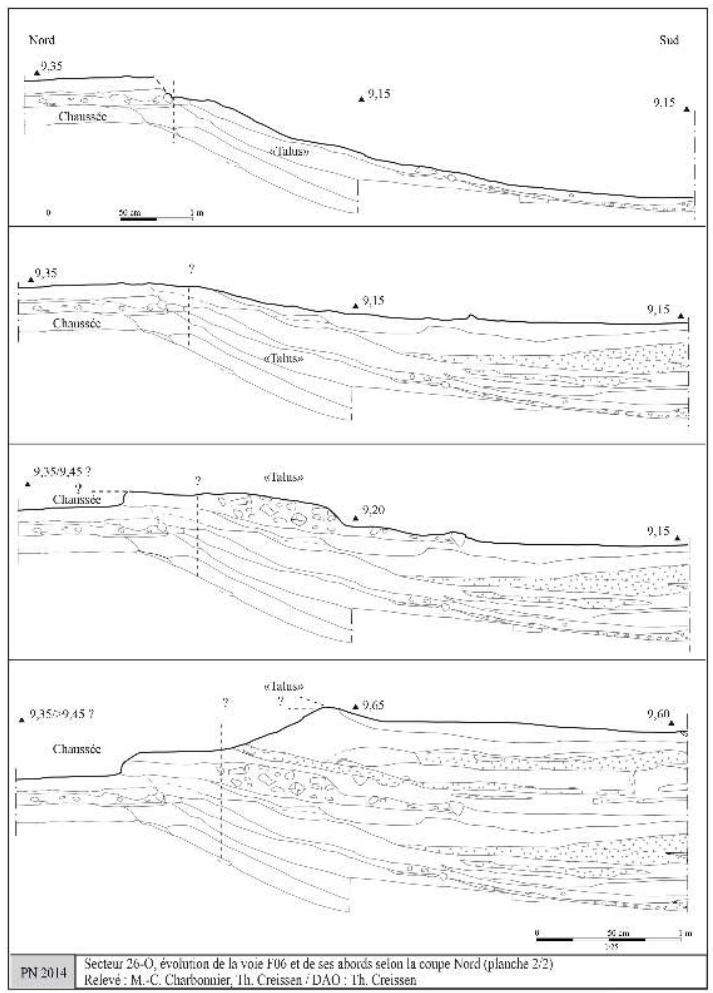

(c) Porta Nocera 2 (M.-C. Charbonnier, Th. Creissen). Sur concession du Ministero per i Beni e le attività culturali - Soprintendenza archeologica di Pompei. Toute reproduction, par quelque moyen que ce soit, reste interdite.

Dans les phases les plus anciennes, la voie se développait au sommet d'un talus, et les monuments funéraires comme l'enclos funéraire précédemment évoqué étaient aménagés dans un terrain situé en contrebas, le dénivelé atteignant au moins $80 \mathrm{~cm}$ dans certains secteurs.

\section{Réfection de la voirie et du talus associé ; mise en place d'un chemin secondaire et absence d'entretien du monument 27 OS}

$16 \mathrm{Au}$ cours du temps, la chaussée comme le talus ont fait l'objet de plusieurs recharges entrainant un élargissement progressif de la voie et une lente atténuation de la pente du talus. Recharges de voirie et réaménagements du talus semblent aller de pair, ce qui donne l'impression d'une gestion planifiée de cet espace. Lors de l'une de ces transformations, un chemin empierré a été aménagé à l'ouest du mausolée 26a. Il se raccordait à la chaussée en passant sur le talus et filait vers le sud est.

$17 \mathrm{Au}$ cours de ces remaniements, les remblais viennent s'accumuler contre le parement ouest du mausolée 27 OS dont le podium est progressivement enseveli. Sur certaines des parties recouvertes, l'enduit peint qui revêt le mausolée avait déjà disparu : il semble que le monument n'était plus guère entretenu. 
Sur ce même enduit, en partie haute, une inscription peinte en rouge a été dégagée, qui pourrait appartenir à cette phase. Malgré son caractère très lacunaire, il est possible d'affirmer que le texte correspond à l'annonce d'un combat de gladiateurs. Le nom de l'évergète comme celui du lieu du combat ne sont plus lisibles. Le choix de cet emplacement se justifie par le fait que le secteur était vierge de constructions, le texte étant alors parfaitement visible depuis la voie principale mais aussi peut-être également depuis le chemin secondaire.

Dans le secteur 26 Ouest, des restes carbonisés ont été retrouvés dans les niveaux correspondant à cette phase. L'étude carpologique a permis d'identifier des restes de fruitiers domestiques (figuier, prunier, olivier, pin pignon). À l'inverse, aucune céréale ou légumineuse n'est présente. Un tel assemblage évoque ce que l'on trouve fréquemment en contexte funéraire. Pour autant, les dépôts de ne sont pas organisés et les vestiges sont en position secondaire: l'ensemble a probablement été déposé de manière plus ou moins anarchique à l'occasion de la fréquentation de la nécropole ou par ruissellement sur la voie et la pente du talus. Les restes se sont alors accumulés dans les parties planes.

\section{Une période d'abandon relatif : accumulation de colluvions, ensevelissement des tombes, ruine du mausolée 26 a et aménagement d'un bûcher}

20 Peu après l'aménagement du chemin empierré, les secteurs situés en contrebas de la voie sont progressivement comblés par un épais remblai multiforme. Ce dernier est constitué d'une alternance de couches limoneuses plus ou moins épaisses et de poches sableuses. L'ensemble a fait l'objet de nombreux prélèvements afin d'en permettre une étude géomorphologique et micro-morphologique. Les analyses seront effectuées par la suite, mais il est d'ores et déjà possible de constater qu'il ne s'agit pas là de l'apport soudain d'un épais remblai, mais de l'accumulation progressive de niveaux de colluvionnement dans une sorte de dépression. Le rythme de cette accumulation reste à déterminer. Au terme de ce processus, l'essentiel du secteur 26 Ouest est désormais plus ou moins à la même altitude que la chaussée.

Ces dépôts donnent l'image d'un secteur assez largement déserté, progressivement comblé par des " coulées de boue ", mais les choses sont très différentes selon que l'on se trouve à l'ouest ou à l'est du mausolée 26a. Dans le secteur 26 Ouest, aucun fait majeur ne s'intercale entre ces séquences d'accumulation. À l'inverse, en partie orientale, il a été possible d'identifier plusieurs structures associées à cette séquence.

L'une d'elle est située dans la partie sud du secteur (F 05; fig. 8). Cette structure a simplement été testée cette année afin d'en préciser la fonction. Dans le sédiment qui la constitue, des esquilles d'os humains brûlés étaient présents. Des bords rubéfiés ont été en partie dégagés. Ces différentes découvertes indiquent qu'il s'agit très certainement d'un bûcher. Il sera fouillé lors de la prochaine campagne par passes successives au sein d'un carroyage de $20 \times 20 \mathrm{~cm}$ implanté dès cette première intervention. 
Fig. 8 - Cliché de l'aire de crémation F 05 en cours de fouille.

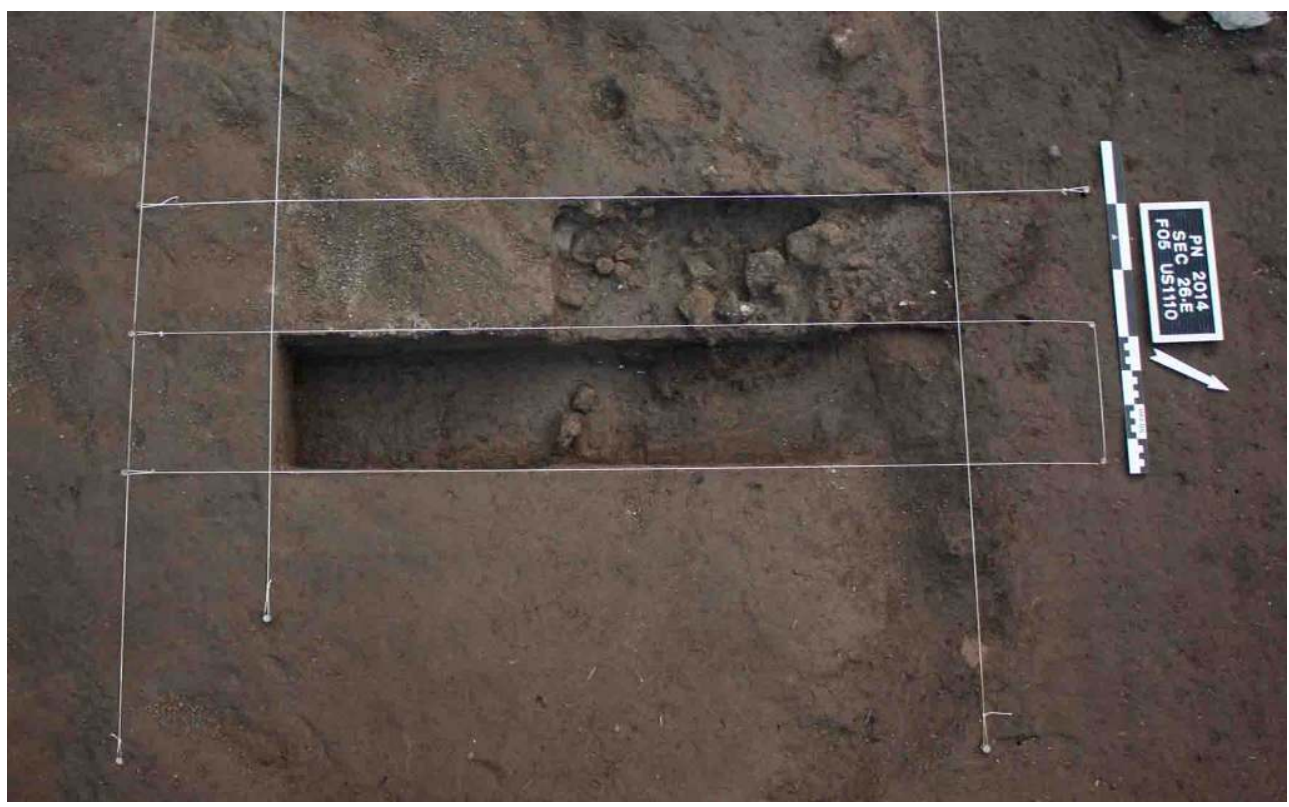

(c) Porta Nocera 2 (G. Sachau-Carcel). Sur concession du Ministero per i Beni e le attività culturali Soprintendenza archeologica di Pompei. Toute reproduction, par quelque moyen que ce soit, reste interdite.

À cette même phase, il faut peut-être rattacher une petite fosse dégagée à la jonction des secteurs 26 Est et $25 \mathrm{C}$ (F 03). Cette dernière est creusée dans un niveau de limon qui scelle les tombes de l'enclos funéraire associé à la phase antérieure. Elle a un diamètre de $35 \mathrm{~cm}$ environ. Son comblement est sableux et mêlé de gravillons. Un fragment osseux brûlé a été identifié en surface : la structure est potentiellement funéraire, et il a donc été décidé de reporter sa fouille à la campagne 2015. Dans cette phase, le monument funéraire 26a est abandonné et en partie ruiné : des pierres provenant de l'effondrement sont associées aux différentes apports de colluvions tout autour du monument.

En l'absence de claires relations stratigraphiques, il faut rappeler que le phasage reste très hypothétique et la contemporanéité de toutes ces structures n'est pas clairement établie. Quant à la datation, l'étude préliminaire de la céramique pourrait indiquer que l'on se situe avant les années 30 à 50 de notre ère.

\section{Réaménagements autour du mausolée ruiné, creusement d'une petite fosse, construction du monument funéraire de Castricia Prisca}

Tout autour du mausolée ruiné, des niveaux essentiellement constitués de matériaux de démolition ont été épandus. À l'ouest, la surface de ce niveau est aplanie. À l'inverse, à l'est, cet épandage suit la pente qui s'était maintenue dans ce secteur. Ces niveaux sont peut-être à rapprocher d'une couche très riche en matériel retrouvée à l'intérieur même du mausolée (elle suit un léger pendage vers les sud). En bordure de la voie, ce niveau passe en partie sur les maçonneries arasées. Parmi le matériel qui y a été retrouvé se distinguent plusieurs fragments d'une même coupe en sigillée marbrée provenant des ateliers de la Graufesenque. 
Une fosse d'un diamètre de $45 \mathrm{~cm}$ environ et profonde d'une petite dizaine de centimètres semble se rattacher à cette phase (F 02). Son comblement était constitué de charbons et d'esquilles d'os, et plusieurs fragments d'un récipient en verre étaient écrasés à sa surface. Ce comblement pourrait correspondre à un rejet de crémation (fig. 9).

Fig. 9 - Cliché de la fosse F 02 en fin de fouille.

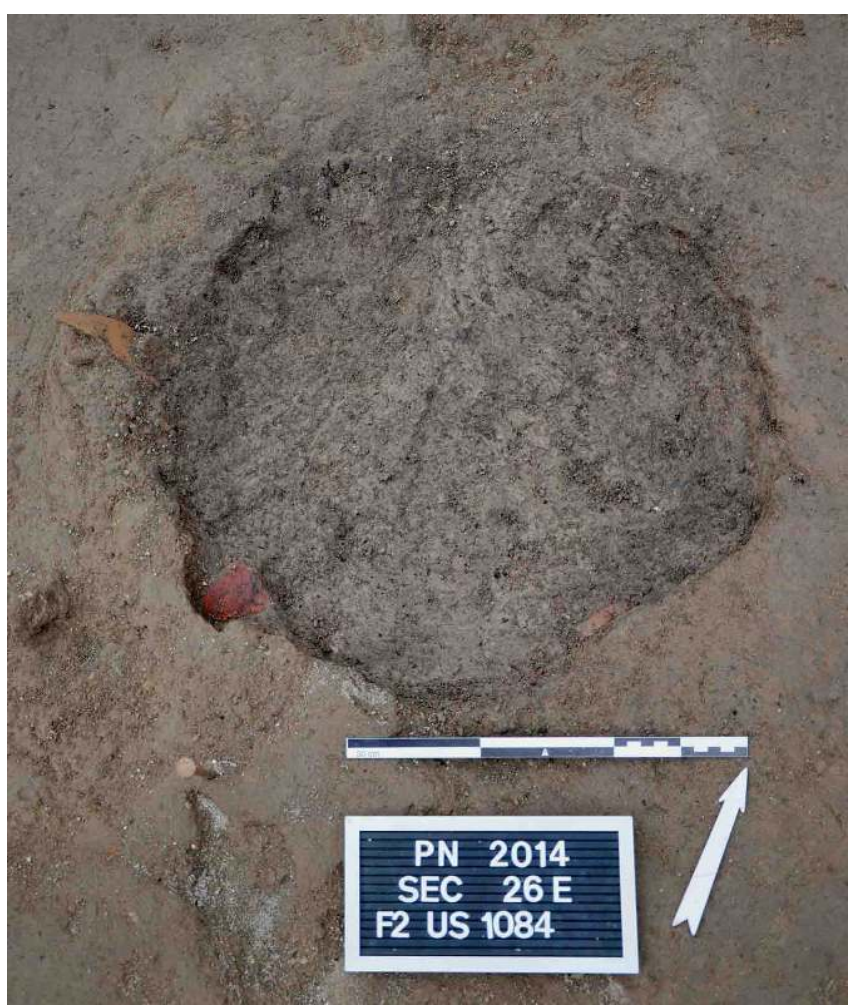

(c) Porta Nocera 2 (G. Sachau-Carcel). Sur concession du Ministero per i Beni e le attività culturali Soprintendenza archeologica di Pompei. Toute reproduction, par quelque moyen que ce soit, reste interdite.

En limite est de la zone étudiée, c'est peut-être à cette phase qu'il faut associer la construction du monument de Castricia Prisca. Il a été construit au dessus des premiers niveaux de colluvions qui envahissent le secteur, alors que l'enclos funéraire antérieur n'était plus visible. Cet ensemble a été étudié au cours des fouilles réalisées entre 2005 et 2007. Le monument est daté des environs de 60 apr. J.-C.

\section{Poursuite de la ruine du mausolée, nouvelles accumulations de niveaux de colluvionnement}

La dernière phase de l'histoire de ce secteur est marquée par la reprise du phénomène de colluvionnement. Des dépôts tantôt limoneux, tantôt sableux continuent à s'accumuler (ils ont fait l'objet de nombreux prélèvements pour étude micro-morphologique). Au terme de ce processus, il semble que ce secteur était maintenant plus élevé que la voie. Il est à vrai dire difficile de comprendre comment ces niveaux ont pu s'accumuler en contre-haut de cette voie : peut-être existait-il une structure permettant de les contenir ou à l'inverse faut-il concevoir que la voie a été curée ? Ces remblais viennent se plaquer contre l'inscription annonçant la tenue du spectacle de gladiateurs. 

en verre est largement brisée et une partie de son contenu se déverse à l'intérieur comme à l'extérieur du monument funéraire. L'étude de ces ossements confirme que tous proviennent de la SEP 301. Un balsamaire en verre de type Isings 8 a été retrouvé au sein du même effondrement. Il semble donc naturel de le rattacher à la tombe, mais la datation de cet élément pose problème : à Pompéi, ce type est plutôt caractéristique de la période tibéro-claudienne, quand la tombe est supposée augustéenne.

D'une manière plus générale, le matériel issu de ces dernières phases de remblai est daté des années 40-70.

\section{L'éruption de 79 apr. J.-C.}

En plusieurs endroits du secteur fouillé, les lapilli contemporains de l'éruption du Vésuve étaient encore en place. Ils scellaient le sommet du niveau de remblai associé à la phase antérieure, lequel recouvrait désormais les parties arasées du mausolée 26a. Ce dernier avait alors totalement disparu du paysage.

En surface de ce remblai, de nombreux petits trous ont été observés, qui paraissent correspondre à des racines. Une dépression plus profonde a été remplie de plâtre ce qui a permis d'identifier la racine d'un arbuste. Dans ce dernier état, le secteur qui borde la voie est désormais vierge de toute construction et il est largement végétalisé.

\section{Le dégagement de la nécropole et les travaux postérieurs}

Pour l'essentiel, les fouilles des années 1950 se sont contentées de dégager les monuments funéraires. À la suite des cette intervention, d'autres travaux sont attestés : il s'agit en particulier de la pose d'une canalisation destinée à l'évacuation des eaux de pluie. À cette occasion, les liens entre les derniers niveaux de la voie et le secteur environnants ont malheureusement été détruits.

\section{La photogrammétrie}

La photogrammétrie a été abondamment mise à profit durant cette campagne. Les principales étapes de la fouille ont été documentées, ce qui permet de restituer de manière précise le déroulement du chantier et l'aspect du terrain avant chaque nouveau démontage (fig. 10 et 11). 
Fig. 10 - Relevé en 3D des US : niveaux antiques après enlèvement des couches modernes.

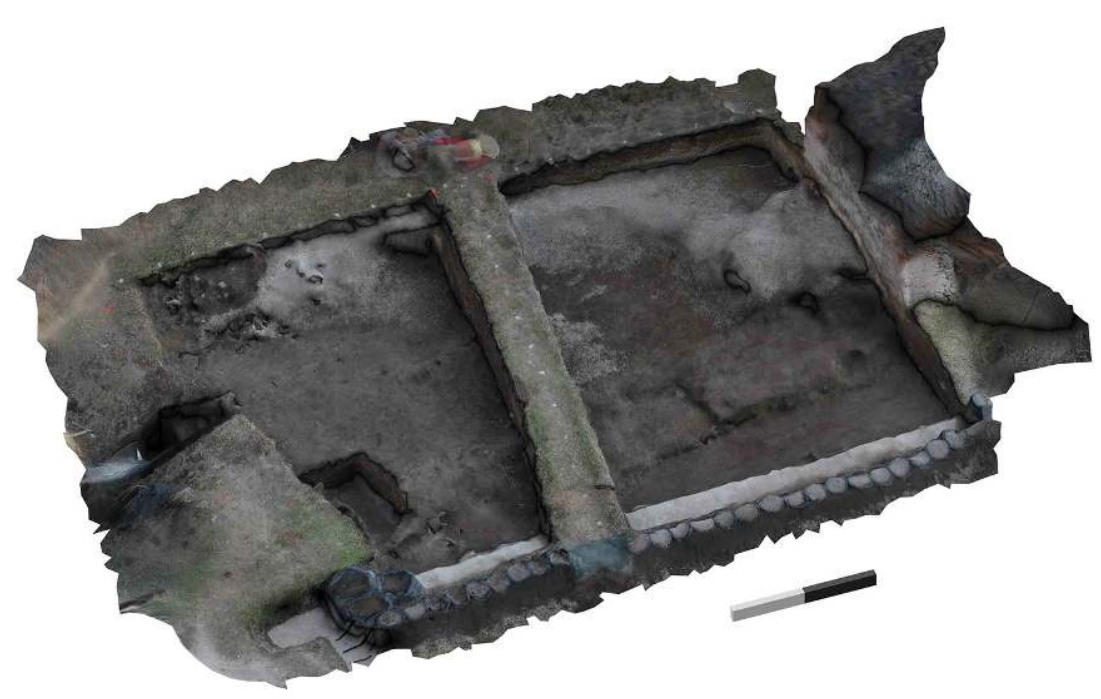

(c) Porta Nocera 2 (G. Sachau-Carcel). Sur concession du Ministero per i Beni e le attività culturali Soprintendenza archeologica di Pompei. Toute reproduction, par quelque moyen que ce soit, reste interdite.

Fig. 11 - Relevé en 3D des US : le monument 26 est progressivement dégagé de sa gangue de colluvions.

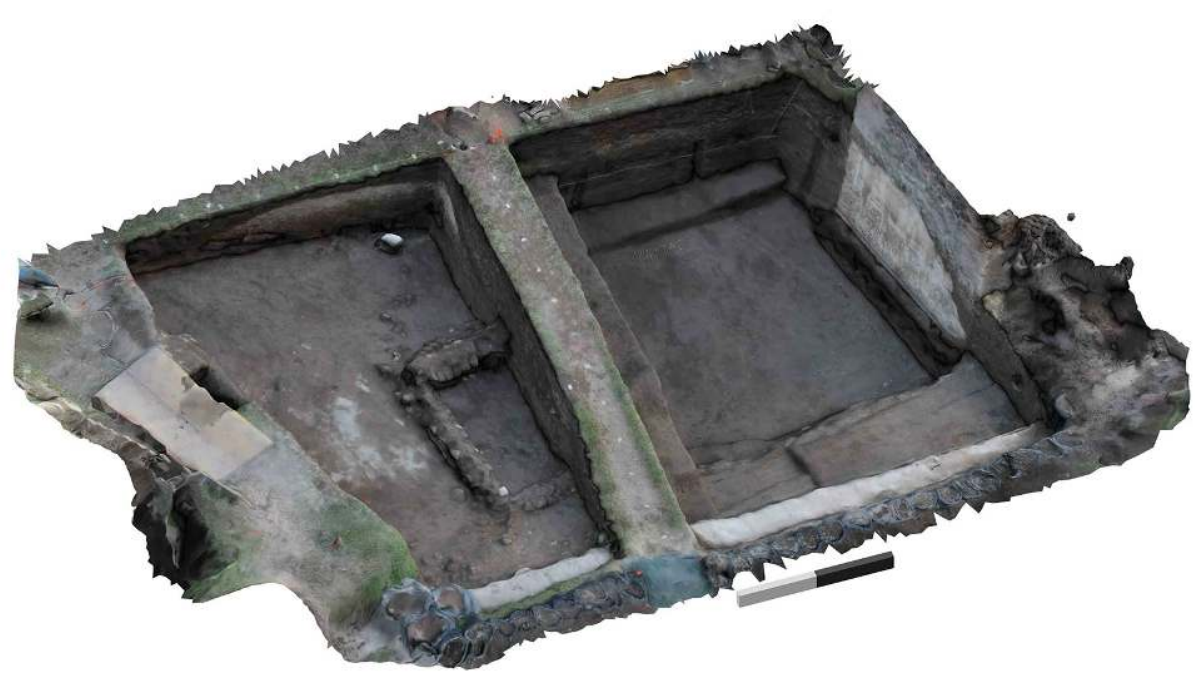

(c) Porta Nocera 2 (G. Sachau-Carcel). Sur concession du Ministero per i Beni e le attività culturali Soprintendenza archeologica di Pompei. Toute reproduction, par quelque moyen que ce soit, reste interdite. 
Une fois combinés, ces documents permettent en outre de générer autant de coupes que jugé nécessaire sans avoir à multiplier les relevés de terrains. Ces documents se révèlent très précieux pour restituer les dynamiques stratigraphiques, mais ils demanderont toutefois à être en partie retraités (fig. 12).

Fig. 12 - Vue nord-sud des profils des différentes passes et unités stratigraphiques enregistrées.

$\mathrm{E}$

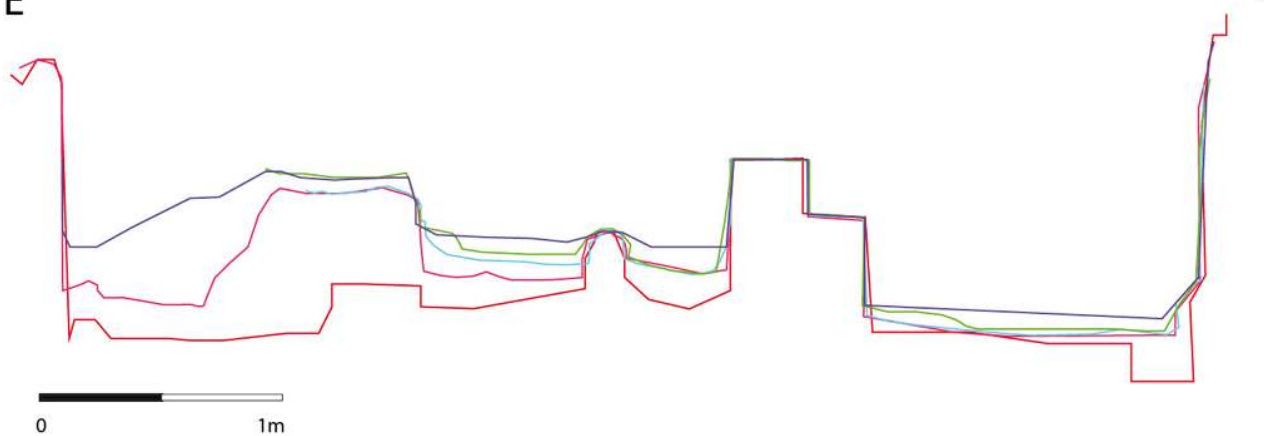

(c) Porta Nocera 2 (G. Sachau-Carcel). Sur concession du Ministero per i Beni e le attività culturali Soprintendenza archeologica di Pompei. Toute reproduction, par quelque moyen que ce soit, reste interdite.

Par ailleurs, la couverture photogrammétrique générale permet là-encore de replacer aisément les zones fouillées dans l'environnement immédiat (fig. 13). Là encore, de grandes coupes peuvent être générées automatiquement (fig. 14). 
Fig. 13 - Vue aérienne des tombeaux 23 à 31 OS de la nécropole de Porta Nocera.

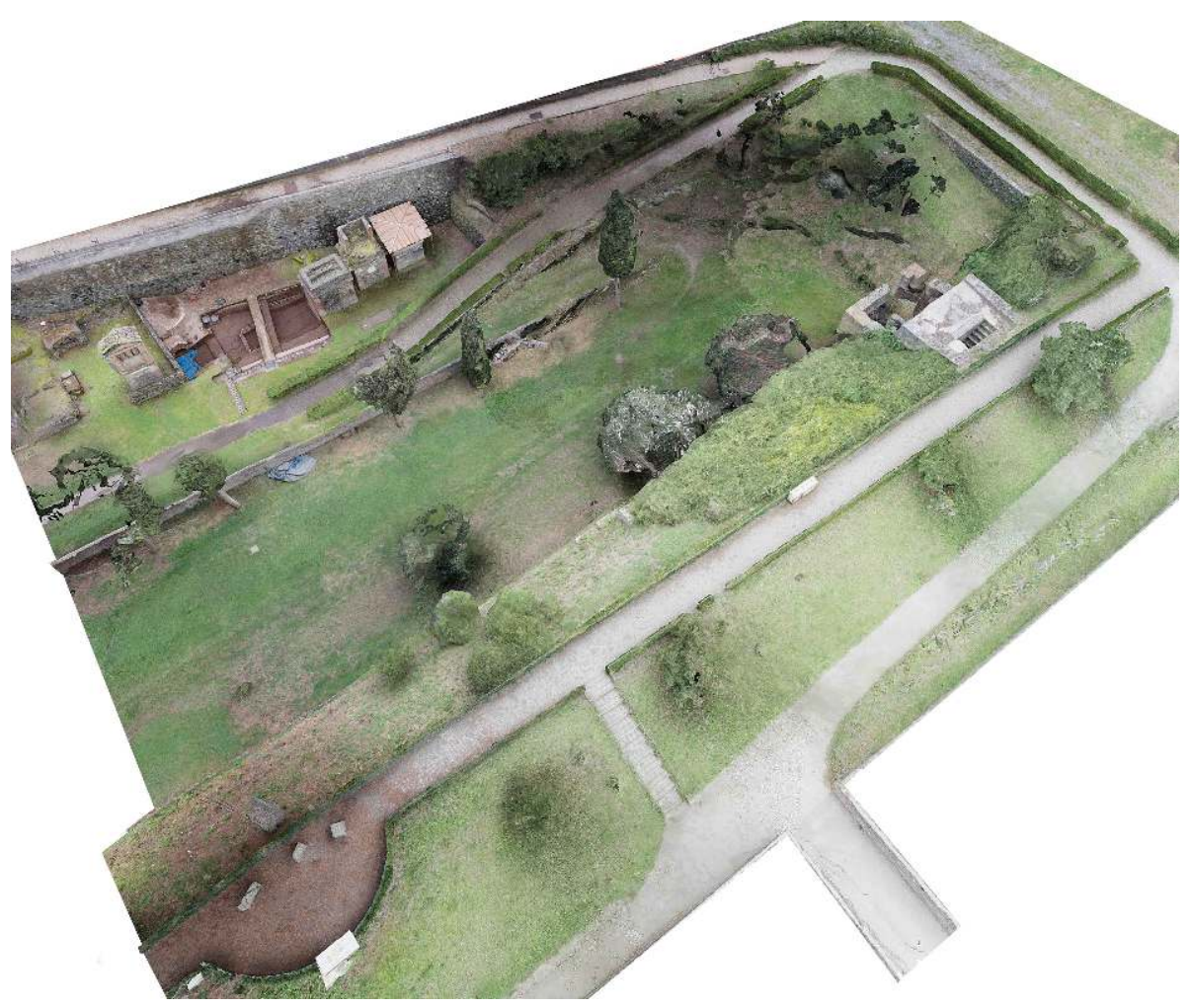

Au premier plan, la muraille.

(c) Porta Nocera 2 (J. Laiho). Sur concession du Ministero per i Beni e le attività culturali - Soprintendenza archeologica di Pompei. Toute reproduction, par quelque moyen que ce soit, reste interdite.

Fig. 14 - Grande coupe Nord-Sud.

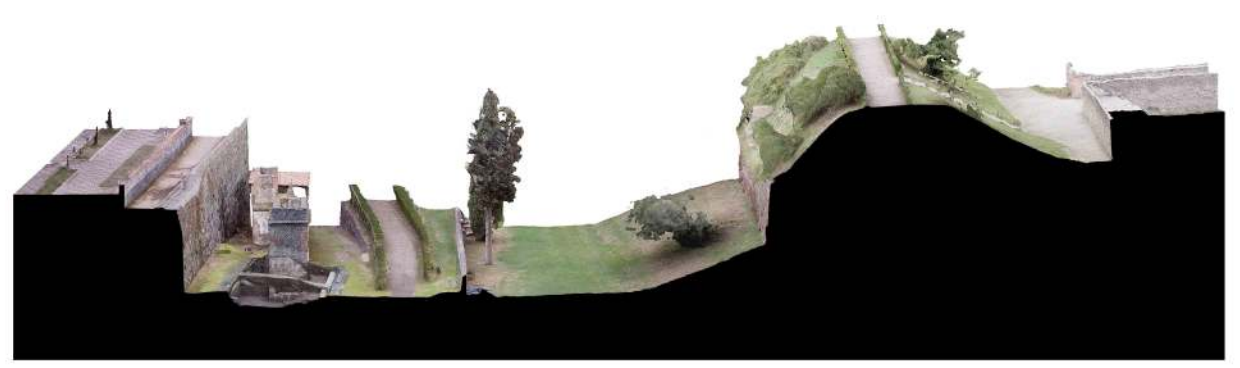

La muraille (à droite) surplombe un fossé qui s'arrête sur un mur. Au-delà, la route et les tombeaux. (c) Porta Nocera 2 (J. Laiho). Sur concession du Ministero per i Beni e le attività culturali - Soprintendenza archeologica di Pompei. Toute reproduction, par quelque moyen que ce soit, reste interdite.

37 La campagne 2014 était prévue comme une campagne préliminaire destinée à affiner les méthodes d'approches en archéologie funéraire et à définir des secteurs d'étude pour les années à venir. Il s'agissait également de construire une équipe qui puisse intervenir dans tous les champs disciplinaires de l'archéologie funéraire. La fouille a permis de dégager une aire funéraire avec quatre tombes (25c), une aire de crémation (F5), une fosse (F3) ainsi qu'un monument funéraire détruit (26a). Ces structures seront fouillées en 2015 et permettront de réfléchir aux dynamiques d'organisation des espaces funéraires. Un autre 
objectif est la fouille du monument 27 OS qui daterait du troisième quart du $\mathrm{I}^{\mathrm{er}}$ siècle av. J.-C. Il s'agirait de mettre en évidence les sols de circulation autour du monument, également de dégager la façade sur rue. Un sondage profond pourra permettre de reconnaître une éventuelle occupation antérieure au monument 27 OS. Ainsi, il sera possible de dresser une carte précise de ce vallon situé à l'ouest de l'affleurement rocheux qui porte les monuments 19, 21 et 23 OS. Cette fouille sera complétée par l'ouverture d'une nouvelle zone de la nécropole qui permettra d'affiner le travail sur les structures et l'activité funéraires (tombes, aires de crémation, nécro-sols).

\section{BIBLIOGRAPHIE}

Van Andringa - Creissen - Duday 2014 = W. Van Andringa, Th. Creissen, H. Duday (dir.) et al., Porta Nocera 2 - Campagne 2014. Fouille de la nécropole romaine de Porta Nocera : le secteur 26 OS [Rapport d'opération], Pompéi, 2014, 250 p.

\section{NOTES}

1. Un rapport d'opération a été déposé auprès des différentes institutions porteuses du programme : Van Andringa - Creissen - Duday 2014.

\section{INDEX}

institutions Programme de recherche de l'École française de Rome en collaboration avec l'université de Lille 3 (Centre de recherches Halma), la société archéologique Éveha International et la Soprintendenza archeologica di Pompei, avec la participation du laboratoire d'anthropologie de Bordeaux, la société Archeodunum et le programme AGRUMED Index géographique : Pompéi

\section{AUTEURS}

\section{WILLIAM VAN ANDRINGA}

Université Lille 3 - william.va[at]free.fr

\section{THOMAS CREISSEN}

Éveha International, Université de Tours - thomas.creissen[at]eveha.fr 


\section{HENRI DUDAY}

CNRS, Université de Bordeaux - h.duday[at]anthropologie.u-bordeaux1.fr 